\title{
Predict Score: A New Biological and Clinical Tool to Help Predict Risk of Intensive Care Transfer for COVID-19 Patients
}

\author{
Mickael Gette ${ }^{1}$, Sara Fernandes ${ }^{2}$, Marion Marlinge 1,3, Marine Duranjou ${ }^{1}$, Wijayanto Adi ${ }^{1}$, Maelle Dambo ${ }^{1}$, \\ Pierre Simeone $^{4}\left(\mathbb{D}\right.$, Pierre Michelet ${ }^{3,5}$, Nicolas Bruder ${ }^{4}$, Regis Guieu ${ }^{1,3, *(D)}$ and Julien Fromonot ${ }^{1,3}$ (D) \\ 1 Laboratory of Biochemistry, Timone University Hospital, APHM, 13005 Marseille, France; \\ mickael.gette@ap-hm.fr or mickaelgette@gmail.com (M.G.); marion.marlinge@ap-hm.fr (M.M.); \\ marine.duranjou@ap-hm.fr (M.D.); maylis.adi-wijayanto@ap-hm.fr (W.A.); maelle.dambo@ap-hm.fr (M.D.); \\ julien.fromonot@univ-amu.fr (J.F.) \\ 2 Center for Research and Studies on Health Services and Quality of Life, Aix-Marseille University, \\ 13005 Marseille, France; sarah.fernandes@etu.univ-amu.fr \\ 3 INSERM, INRAE, C2VN, Aix-Marseille University, 13005 Marseille, France; pierre.michelet@univ-amu.fr \\ 4 Department of Anesthesiology and Intensive Care, Timone University Hospital, \\ Aix Marseille University APHM, 13005 Marseille, France; pierre.simeone@ap-hm.fr (P.S.); \\ nicolas.bruder@ap-hm.fr (N.B.) \\ 5 Department of Emergency Medicine and Intensive Care, Timone University Hospital, APHM, \\ 13005 Marseille, France \\ * Correspondence: guieu.regis@orange.fr
}

check for updates

Citation: Gette, M.; Fernandes, S.; Marlinge, M.; Duranjou, M.; Adi, W.; Dambo, M.; Simeone, P.; Michelet, P.; Bruder, N.; Guieu, R.; et al. Predict Score: A New Biological and Clinical Tool to Help Predict Risk of Intensive Care Transfer for COVID-19 Patients. Biomedicines 2021, 9, 566. https:/ / doi.org/10.3390/biomedicines 9050566

Academic Editor: Francesco Ceci

Received: 28 March 2021

Accepted: 29 April 2021

Published: 18 May 2021

Publisher's Note: MDPI stays neutral with regard to jurisdictional claims in published maps and institutional affiliations.

Copyright: (c) 2021 by the authors. Licensee MDPI, Basel, Switzerland. This article is an open access article distributed under the terms and conditions of the Creative Commons Attribution (CC BY) license (https:// creativecommons.org/licenses/by/ $4.0 /)$

\begin{abstract}
Background: The COVID-19 crisis has strained world health care systems. This study aimed to develop an innovative prediction score using clinical and biological parameters (PREDICT score) to anticipate the need of intensive care of COVID-19 patients already hospitalized in standard medical units. Methods: PREDICT score was based on a training cohort and a validation cohort retrospectively recruited in 2020 in the Marseille University Hospital. Multivariate analyses were performed, including clinical, and biological parameters, comparing a baseline group composed of COVID-19 patients exclusively treated in standard medical units to COVID-19 patients that needed intensive care during their hospitalization. Results: Independent variables included in the PREDICT score were: age, Body Mass Index, Respiratory Rate, oxygen saturation, C-reactive protein, neutrophil-lymphocyte ratio and lactate dehydrogenase. The PREDICT score was able to correctly identify more than $83 \%$ of patients that needed intensive care after at least 1 day of standard medical hospitalization. Conclusions: The PREDICT score is a powerful tool for anticipating the intensive care need for COVID-19 patients already hospitalized in a standard medical unit. It shows limitations for patients who immediately need intensive care, but it draws attention to patients who have an important risk of needing intensive care after at least one day of hospitalization.
\end{abstract}

Keywords: COVID-19; score; biology; intensive care

\section{Introduction}

In December 2019, medical teams of Wuhan, Hubei, China discovered a novel coronavirus responsible for acute respiratory distress syndrome (ARDS). They were able to identify this new pathogen using next-generation sequencing and transforming the real-time polymerase chain reaction (RT-PCR) in a "user-friendly" diagnostic tool for laboratories with little familiarity with this technology [1].

The severe acute respiratory syndrome coronavirus 2 propagated around the world until it was declared a Worldwide Public Health Emergency on the 30th of January 2020 by the World Health Organization (WHO), being considered a threat to health care systems [2]. The WHO emergency committee recommended massive detection strategies, isolation of contaminated patients, early treatment and new technological contact-tracing systems to limit the spread of COVID-19. However, several months after the beginning of this 
pandemic, two facts remain constant: the lack of resources, and that isolation has been the only effective strategy in limiting the spread of the disease.

Hospitals had to adapt to this new situation daily, restricting their access to non-urgent diseases, increasing the number of beds in their intensive care unit (ICU), and isolating COVID patients despite the lack of adequate protective equipment (qualitatively and quantitatively) for health workers and non-COVID patients [3].

In France, 89,818 patients were hospitalized, of which 4387 required intensive care and 23,686 died from COVID between 1 March 2020 and 28 April 2021 [4]. In the Provence Alpes Cote d'Azur Region, during the same period, 1220 patients were hospitalized, of which 295 needed intensive care treatment, and 82 died from COVID [5].

Modern medical biology has the potential to acquire an important role in this type of crisis, as it is indispensable for diagnosis and useful for the development of a treatment plan and guiding medical decisions and hospitalization scheduling [6-8]. Previous studies have identified biomarkers that significantly document a high risk of progression to severe forms of COVID-19 [9], such as interleukin-6 and D-Dimer levels. Others have proposed the use of a composite risk score [10-12], using clinical data similarly to the National Early Warning Score 2 (NEWS 2), medical history and different biomarkers, but requiring a web calculator.

The aim of this study was to create a composite risk score using biological and clinical parameters, that evaluated the risk of COVID-19-positive patients hospitalized in a Standard Medical Unit (SMU) needing intensive care during the days following hospitalization. Thus, helping medical teams anticipate the level of medical care a patient will need, and therefore allowing them to use their resources wisely, particularly ICU beds and artificial respirators. During this study, the main preoccupation was to build a user-friendly score, using biological parameters which are widely available throughout the world, easily measured clinical parameters and the patient's intrinsic constants, without neglecting discrimination capacity.

\section{Materials and Methods}

\subsection{Study Design and Patient Selection}

We designed a retrospective monocentric study, including all health care centers of the Assistance Publique des Hôpitaux de Marseille (AP-HM) (Public Assistance of Marseille Hospitals), France. Biological resources, medical imaging and clinical records were all produced in different AP-HM sites.

From 29 February 2020 to 30 April 2020, all adult patients diagnosed with COVID-19 according to WHO guidelines [13] were initially included in a first cohort (see Figure 1). Patient selection did not consider patients' characteristics, age, sex, medical history, treatments, or initial clinical evaluation and vital signs. This first cohort was used to construct the PREDICT score (predicting risk factors for early determination of ICU transfer). A second cohort of patients was enrolled, from 1 August to 25 October 2020, using the same criteria as previously, to validate the score.

The subjects were separated into three different groups, based on disease severity and their requirement for intensive care:

1. Patients admitted to the Standard Medical Unit were included in the SMU group;

2. Patients admitted directly into the Intensive Care Unit directly were included in the ICU group;

3. Patients that initially were admitted to the Standard Medical Unit for at least $24 \mathrm{~h}$, but subsequently needed to be transferred to the Intensive Care Unit were included in a third group, named Standard to Intensive Care (STol) group.

Two reasons motivated this choice: firstly the need for intensive care is associated with complications which are unmanageable by a standard medical unit (SMU), secondly the limited number of places in Intensive Care Unit (ICU) confers a critical value to their management. During their practice, physicians employed general severity tools for respiratory diseases and used their clinical judgement to decide when patients needed intensive 
care unit $[14,15]$, as stated in international guidelines and recent recommendations, but no specific scores.

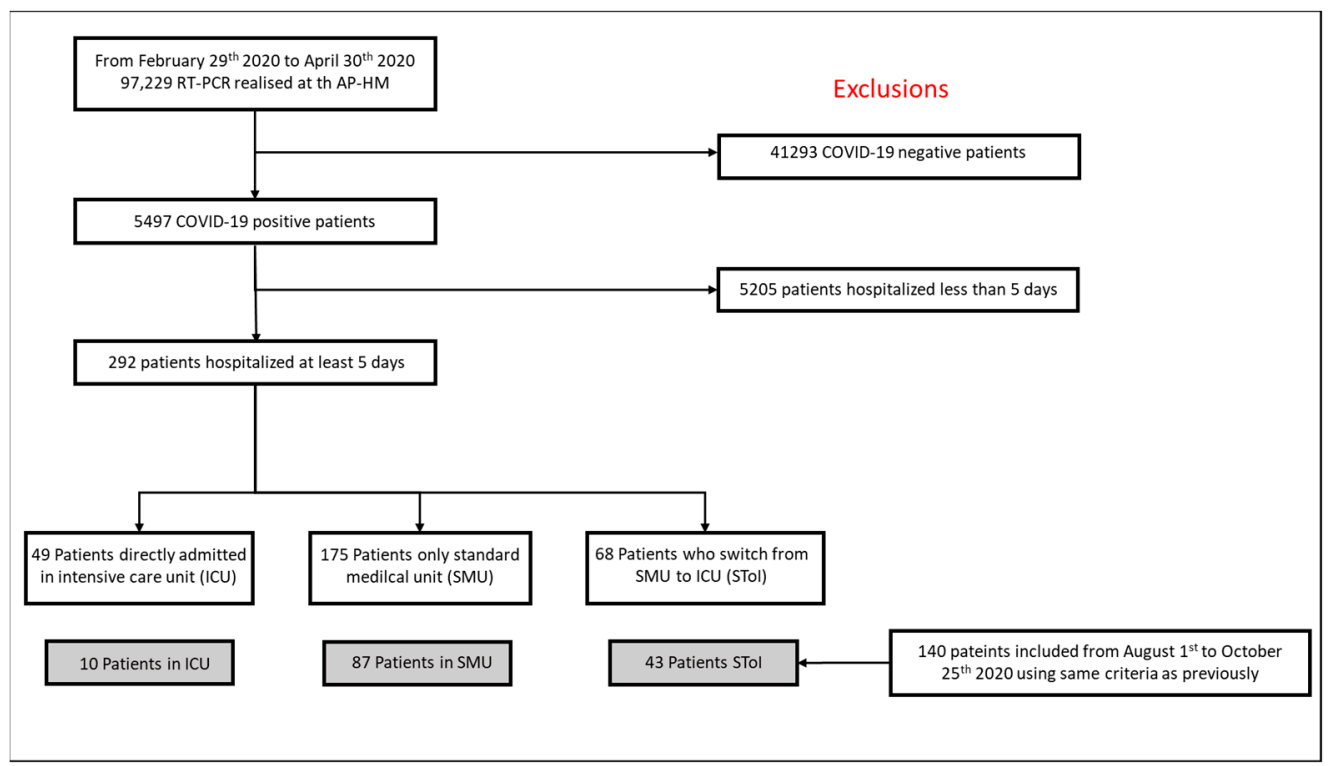

Figure 1. Flow chart.

In the training cohort, 175 patients were admitted to standard medical unit (SMU group), 49 patients were admitted to the intensive care unit directly (ICU group) and 68 patients were initially admitted in a standard medical unit but later required intensive care (STol group). In the validation cohort, 87 were included in the SMU group, 10 patients in the ICU group, and 43 patients in the SToI group.

To train the PREDICT score, after having analyzed a lot of parameters (intrinsic, comorbidity, vital sign, biologic) and a lot of combinations of these, a multivariate analysis highlighted a significative combination of age, Body Mass Index, oxygen saturation $\left(\mathrm{SpO}_{2}\right)$ at admission, respiratory rate at admission, neutrophil-lymphocyte ratio, C-reactive protein and lactate dehydrogenase.

\subsection{Exclusion Criteria}

Patients with fewer than 5 days of hospitalization were excluded, to be coherent with virologic load following, given that previous studies reported that the median time of RT-PCR ending detection was 10 days [16] and, in the two cohorts, median time between first symptoms and hospitalization was 5 days.

Furthermore, patients who stayed less than 5 days in hospital were suffering from a low-severity form of COVID-19 and are beyond the scope of this study.

\subsection{Clinical, Imaging and Laboratory Data Collection}

Axigate software was used to collect clinical data from medical records, like vital sign monitoring (body temperature $\left[\mathrm{T}^{\circ} \mathrm{C}\right]$, cardiac and respiratory frequency, oxygen saturation $\left[\mathrm{SpO}_{2}\right]$, systolic and diastolic blood pressure), symptomatology at admission (fever, dyspnea, cough, anosmia, ageusia, digestive troubles), oxygen requirement, height, weight, body-mass index, and past medical history. Oxygen saturation $\left(\mathrm{SpO}_{2}\right)$ was evaluated before oxygen therapy in all cases.

Furthermore, important dates were recorded, such as the day of symptom commencement, first day of hospitalization, changeover date to ICU if required, and release date from ICU. Further, medical progress notes were collected, and important features such as endotracheal intubation and acute respiratory distress symptoms were recorded. Regarding imaging, radiologic reports of unenhanced low-dose chest-computed tomography 
were used; the AP-HM imaging unit uses a standardized report with qualitative appreciation lung damage sorted into four levels: Absence, Minor, Intermediary, Severe. Finally, laboratory data were collected with the Nextlab Software used by both AP-HM laboratories.

\subsection{Laboratory Findings}

Based on previous studies and considering our aim to use only common parameters, we decided to collect data on natremia (Na), C-reactive protein (CRP), ferritinemia (FRT), lactate dehydrogenase (LDH), creatinine (CREAT), total bilirubin (BILI), aspartate aminotransferase (ASAT), and alanine aminotransferase (ALAT). Biochemical parameters were measured with a COBAS C701 provided by Roche Hitachi, and all reagents used came from Roche (Meylan, France).

Additionally, lymphocyte count (LY) and neutrophils cells count (NEU) were recorded to use the neutrophil-lymphocyte ratio (NLR), as a significant biomarker; platelet count was also included. These analyses were performed by a XN-3000 provided by Sysmex. D-Dimer and fibrinogen measures were also recorded, performed on a Star Max provided by Stago; reagents were provided by Stago as well (Stago Canada, Ltée).

A raw laboratory parameter database was created to record this information, allowing for kinetic-follow up of each parameter for each patient.

\subsection{Definitions}

To evaluate the clinical severity at admission, the NEWS 2 was used, which includes heart and respiration rate, oxygen saturation and supplementation, systolic blood pressure, consciousness, temperature and age [17]. It is an easy way to classify patient severity, helping medical teams treat their patients correctly. Further, age has been reported as an independent risk factor for disease severity $[18,19]$, with a threshold at 65 years. Radiological severity was defined as Minor when patients had 3 compromised sites, with 3 lobules affected on each site (maximum 9 lobules); Intermediary, when patients had a minimum of 10 lobules affected, but less than $50 \%$ of total segmental volume; Severe, when more than $50 \%$ of total segmental volume was affected. Acute respiratory failure (ARF) was defined as respiratory rate $>20$ (or accessory muscle use for ventilation), and hypoxemia (oxygen partial pressure (PaO2) lower than $60 \mathrm{~mm} \mathrm{Hg}$ on breathing room air), acute respiratory distress syndrome (ARDS, Berlin definition); acute respiratory failure not explained by cardiac failure or fluid overload with bilateral lung opacities on chest imaging and $\mathrm{PaO} 2 / \mathrm{FiO} 2<300$ with positive end-expiratory pressure $>5 \mathrm{~cm} \mathrm{H} 2 \mathrm{O}$ (Fraction of inspired oxygen: FiO2). [20].

\subsection{Statistical Analysis}

Two cohorts were analyzed: the training cohort $(N=292)$ and the validation cohort $(n=140)$. The baseline patient characteristics were expressed as frequencies and percentages for categorical variables and as mean \pm standard deviation or as median and interquartile ranges for continuous variables. First, three comparisons were performed between groups: SMU vs. ICU, SMU vs. StoI, and ICU vs. StoI. The Shapiro-Wilk test was applied to assess the normality of the data. Continuous variables were compared using Mann-Whitney U-test; categorical variables were compared using the Chi-square test or Fisher's exact test, as appropriate. The comparisons were performed between groups within each cohort.

Second, to compare the kinetics of the biological parameters over time between the three groups, we performed separate linear mixed model (LMM) analysis for 14 biological parameters collected at different times. We also performed univariate logistic regressions to identify which clinical parameters were significantly associated with the likelihood of being transferred to an intensive care unit. For easier application to the prediction score model, significant continuous parameters were then converted to categorical variables according to the optimal cutoff value derived from the Youden index (C-reactive protein (CRP), lactate dehydrogenase (LDH), neutrophil-lymphocyte ratio (NLR), peripheral oxygen saturation 
$\left(\mathrm{SpO}_{2}\right)$ and respiratory rate). Body mass index $\geq 30 \mathrm{~kg} / \mathrm{m}^{2}$ and age $<75$ years were identified as risk factors for ICU transfer. The covariates included were: time, temperature, $\mathrm{SpO}_{2}$, Respiratory rate, age, Body Mass Index, sex and comorbidities (diabetes, hypertension, cardio-vascular diseases, dyslipidemia, chronic obstructive pulmonary disease, asthma, tobacco, active and remission cancer, kidney disease).

Third, a score to predict the need for transfer to an intensive care unit was constructed using the training cohort by performing a multivariate logistic regression analysis. The dependent variable was transfer to ICU (yes-no); eigh independent variables (age, body mass index, respiratory rate, oxygen saturation, neutrophil-lymphocyte ratio at admission and in follow-up, CRP in follow-up, LDH in follow-up, and time) were entered in the model. The multivariate regression coefficients were used to assign integer points for the prediction score; each coefficient was multiplied by two and rounded to the nearest integer. Individual risk estimates were based on the sum of weighted scores for each variable; the in-hospital time was time-weighted to identify patients at low risk of being transferred to an intensive care unit. Results were presented as odd ratios and their $95 \%$ confidence intervals (CIs). The PREDICT score was subsequently tested on the validation cohort.

Fourth, the PREDICT score was calculated at three different times: admission, day 1 , and day 2. For each score, area under the receiver operating characteristic (ROC) curves and the Youden index were calculated. Youden index is defined for all point of ROC curves (sensitivity + specificity -1 ) and the maximum value of this index was selected to be the optimal cut-off point and name Youden's threshold. Sensitivity, specificity, positive predictive values, and negative predictive values were provided as percentages and their respective $95 \%$ CIs.

Fifth, the biological parameters were compared between the groups: at each time (Student's $t$-test or Mann-Whitney test) and globally, on the different evaluation times (generalized linear models). A $p$ value $<0.05$ was considered statistically significant. All statistical analyses were performed using SPSS version 20.0 (IBM, Armonk, NY, USA).

Informed consent was obtained from all subjects involved in the study.

\section{Results}

\subsection{Patient Characteristics}

Univariate analysis between SMU vs. ICU groups and SMU vs. STol groups, identified that patients with ages inferior to 75 years were more likely to be admitted to ICU (Odd Ratio 2.3 (IC 95\%: 1.03-5.1; $p=0.0481$ ) and Odd Ratio 2.3 (IC 95\%: 1.2-4.3; $p=0.005$ ), respectively). Tables $1-4$. Table 5 (and Table S1) represents the method calculation of the NEWS2 score. 


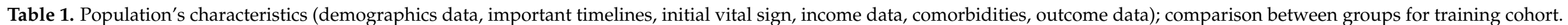

Parameters with a $p$-value $<0.05$ have significative differences between groups compared. ${ }^{*} p<0.05$; ${ }^{* *} p<0.01 ;{ }^{* * *} p<0.001$.

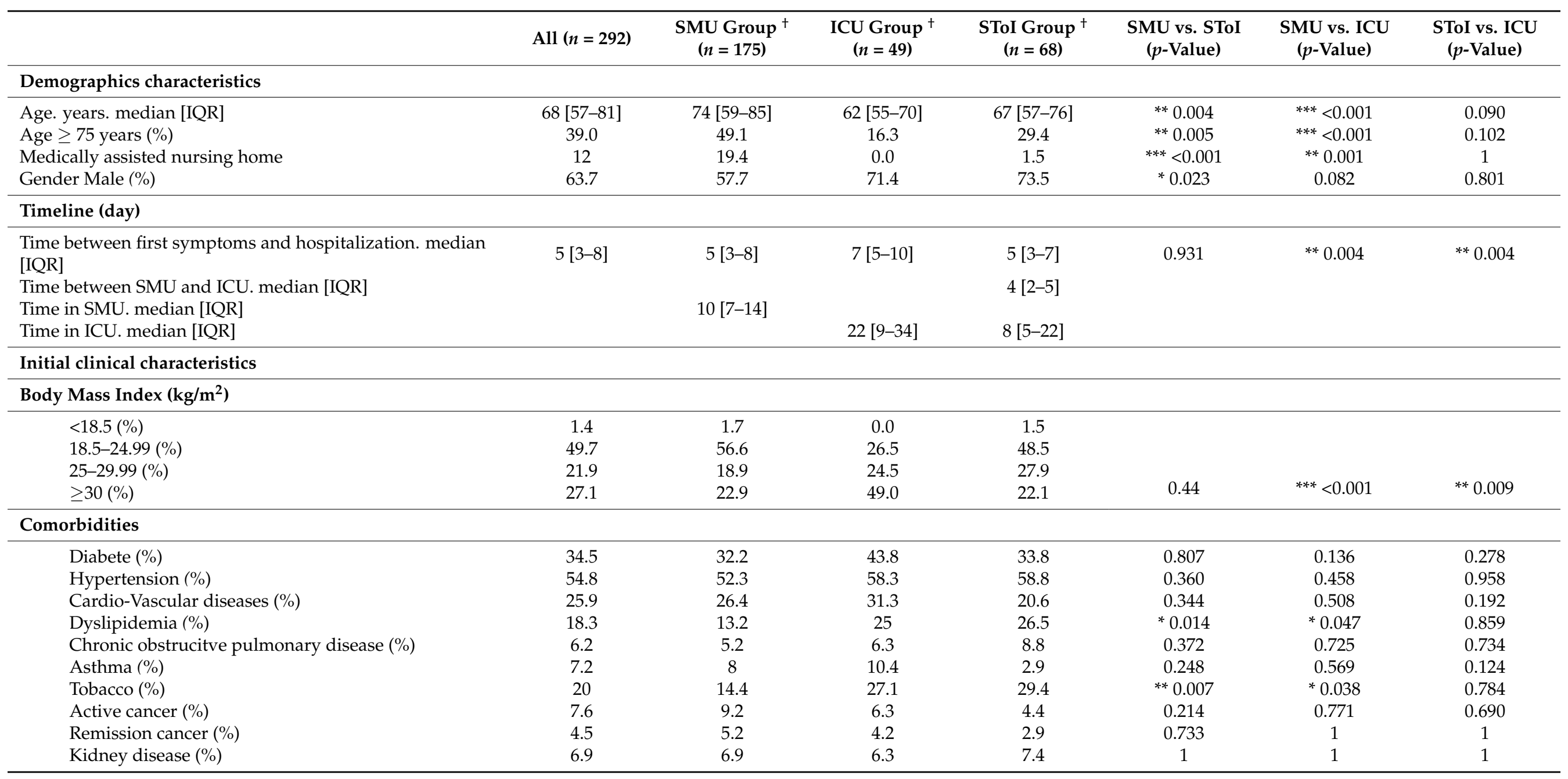


Table 1. Cont.

\begin{tabular}{|c|c|c|c|c|c|c|c|}
\hline & All $(n=292)$ & $\begin{array}{l}\text { SMU Group }^{\dagger} \\
\quad(n=175)\end{array}$ & $\begin{array}{l}\text { ICU Group }^{\dagger} \\
\quad(n=49)\end{array}$ & $\begin{array}{l}\text { SToI Group }^{\dagger} \\
\quad(n=68)\end{array}$ & $\begin{array}{l}\text { SMU vs. SToI } \\
\quad(p \text {-Value })\end{array}$ & $\begin{array}{l}\text { SMU vs. ICU } \\
\text { (p-Value) }\end{array}$ & $\begin{array}{c}\text { SToI vs. ICU } \\
\text { (p-Value) }\end{array}$ \\
\hline \multicolumn{8}{|l|}{ Symptoms on admission } \\
\hline Dyspnea (\%) & 52.9 & 37.4 & 95.9 & 61.8 & ** 0.001 & $* * *<0.001$ & $* * *<0.001$ \\
\hline Fever $(\%)$ & 74.6 & 70.7 & 75.5 & 83.8 & $* 0.035$ & 0.508 & 0.264 \\
\hline Cough $(\%)$ & 55,0 & 51.7 & 51.0 & 66.2 & *0.042 & 0.931 & 0.099 \\
\hline Ageusia-Anosmia (\%) & 16.2 & 14.9 & 10.2 & 23.5 & 0.113 & 0.397 & 0.064 \\
\hline Diarrhea (\%) & 19.2 & 16.7 & 16.3 & 27.9 & *0.048 & 0.955 & 0.141 \\
\hline Heart rate. median [IQR] & 90 [79-101] & $89[78-100]$ & $94[81-102]$ & 90 [79-102] & 0.344 & 0.126 & 0.511 \\
\hline Respiratory rate. median [IQR] & $24[19-28]$ & $22[18-26]$ & $30[25-35]$ & $24[20-30]$ & * 0.021 & $* * *<0.001$ & $* * *<0.001$ \\
\hline Systolic blood pressure. median [IQR] & $132[120-150]$ & $133[120-150]$ & 126 [119-143] & $130[114-145]$ & 0.23 & 0.132 & 0.678 \\
\hline Distolic blood pressure. median [IQR] & $74[63-83]$ & $74[64-82]$ & $70[61-83]$ & $75[63-87]$ & 0.677 & 0.291 & 0.261 \\
\hline Temperature. median [IQR] & $37.4[36.8-38.3]$ & $37.1[36.7-38]$ & $38.1[37.1-38.8]$ & $37.9[37-38.5]$ & $* 0.04$ & $* * *<0.001$ & 0.265 \\
\hline Oxygen saturation (Sp O2) median [IQR] & 95 [93-97] & 96 [93-97] & 94 [89-95] & 95 [93-96] & *0.036 & $* * *<0.001$ & $* * *<0.001$ \\
\hline NEWS-2. median [IQR] & $5[2-7]$ & $4[2-5]$ & $7[6-8]$ & $5[3-7]$ & ** 0.002 & $* * *<0.001$ & $* * *<0.001$ \\
\hline Low risk $(\%)$ & 48.1 & 61,0 & 5 & 43.1 & & & \\
\hline High risk $(\%)$ & 23.9 & 11.3 & 62.5 & 30.8 & & & \\
\hline
\end{tabular}

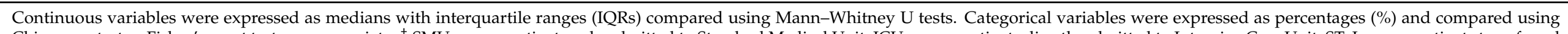

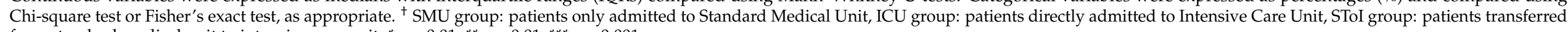
from standard medical unit to intensive care unit. ${ }^{*} p<0.01$ : ${ }^{* *} p<0.01$; ${ }^{* * *} p<0.001$ 


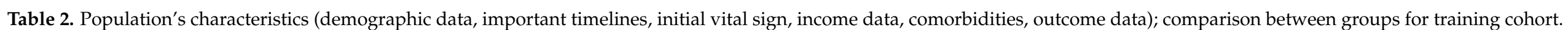

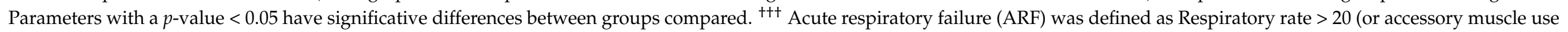

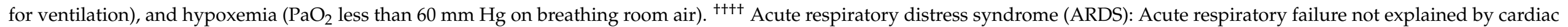

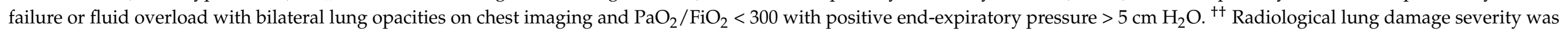

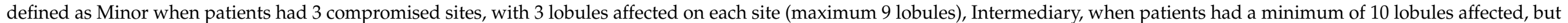

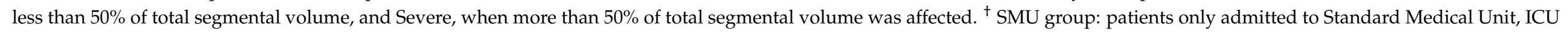
group: patients directly admitted to Intensive Care Unit, SToI group: patients transfered from Standard medical unit to Intensive care unit. ${ }^{*} p<0.05$; ${ }^{* *} p<0.01$; ${ }^{* * *} p<0.001$.

\begin{tabular}{|c|c|c|c|c|c|c|c|}
\hline & All $(n=292)$ & $\begin{array}{l}\text { SMU Group }^{\dagger} \\
\quad(n=175)\end{array}$ & $\begin{array}{c}\text { ICU Group }^{\dagger} \\
(n=49)\end{array}$ & $\begin{array}{l}\text { SToI Group }^{\dagger} \\
\quad(n=68)\end{array}$ & $\begin{array}{l}\text { SMU vs. SToI } \\
\text { (p-Value) }\end{array}$ & $\begin{array}{l}\text { SMU vs. ICU } \\
\text { (p-Value) }\end{array}$ & $\begin{array}{c}\text { SToI vs. ICU } \\
\text { ( } p \text {-Value) }\end{array}$ \\
\hline \multicolumn{8}{|l|}{ Initial O2 needed } \\
\hline Yes $(\%)$ & 36.5 & 35.8 & 73.8 & 42.6 & ** 0.008 & $* * *<0.001$ & $* * 0.001$ \\
\hline Volume. median [IQR] & $4[3-9]$ & $3[2-5]$ & $12[5-15]$ & $3[2-6]$ & 0.443 & $* * *<0.001$ & $* * *<0.001$ \\
\hline \multicolumn{8}{|l|}{ Computed tomography (CT) low dose COVID-19 } \\
\hline Yes $(\%)$ & 94.2 & 98.3 & 75.5 & 97.1 & 0,622 & $* * *<0.001$ & $* * *<0.001$ \\
\hline Absence $(\%)$ & 6.9 & 10.4 & 0.0 & 1.6 & \multirow{4}{*}{$* * * 0.001$} & \multirow{4}{*}{$* * *<0.001$} & \multirow{4}{*}{$* * *<0.001$} \\
\hline Minor $(\%)$ & 22.5 & 29.9 & 2.9 & 14.1 & & & \\
\hline Intermediary (\%) & 39.3 & 42.7 & 11.8 & 45.3 & & & \\
\hline Severe $(\%)$ & 31.4 & 17.1 & 85.3 & 39.1 & & & \\
\hline \multicolumn{8}{|l|}{ Outcomes } \\
\hline Pulmonary embolism (\%) & 4.8 & 2.3 & 8.2 & 8.8 & ${ }^{*} 0.031$ & 0.071 & 1 \\
\hline Cerbebral strocke $(\%)$ & 1.7 & 0.6 & 6.1 & 1.5 & 0.482 & *0.034 & 0.307 \\
\hline Deep vein thrombosis (\%) & 7.2 & 1.7 & 22.4 & 10.3 & *0.06 & $* * *<0.001$ & 0.072 \\
\hline Azithomycin $(\%)$ & 91.4 & 94.3 & 79.6 & 92.6 & 0.767 & $* * 0.003$ & ${ }^{*} 0.037$ \\
\hline Hydroxychloroquine (\%) & 56.2 & 49.1 & 61.2 & 70.6 & $* * 0.003$ & 0.135 & 0.289 \\
\hline${ }^{++\dagger}$ Acute repiratory failure $(\%)$ & 47.3 & 12.6 & 100 & 98.5 & $* * *<0.001$ & $* * *<0.001$ & 1 \\
\hline${ }^{+++\dagger}$ Acute respiratory distress syndrome [ARDS] (\%) & 37.7 & 5.1 & 98 & 77.9 & $* * *<0.001$ & $* * *<0.001$ & $* * 0.002$ \\
\hline Death $(\%)$ & 16.8 & 14.3 & 20.4 & 20.6 & 0.230 & 0.297 & 0.981 \\
\hline Maximum $\mathrm{O} 2$ help & & & & & & & \\
\hline
\end{tabular}


Table 2. Cont.

\begin{tabular}{|c|c|c|c|c|c|c|c|}
\hline & All $(n=292)$ & $\begin{array}{l}\text { SMU Group }^{+} \\
(n=175)\end{array}$ & $\begin{array}{c}\text { ICU Group }^{\dagger} \\
(n=49)\end{array}$ & $\begin{array}{c}\text { SToI Group }^{+} \\
\quad(n=68)\end{array}$ & $\begin{array}{l}\text { SMU vs. SToI } \\
\text { (p-Value) }\end{array}$ & $\begin{array}{l}\text { SMU vs. ICU } \\
\text { (p-Value) }\end{array}$ & $\begin{array}{l}\text { SToI vs. ICU } \\
\text { ( } p \text {-Value) }\end{array}$ \\
\hline \multicolumn{8}{|l|}{ High-concentration mask } \\
\hline Yes $(\%)$ & 9.6 & 0 & 16.3 & 30.9 & $* * *<0.001$ & $* * *<0.001$ & \\
\hline O2 Volume (L/min). Median [IQR] & $30[15-50]$ & NA & $40[28-50]$ & $30[15-50]$ & $* * *<0.001$ & $* * *<0.001$ & 0.518 \\
\hline \multicolumn{8}{|l|}{ Oro-tracheal intubation } \\
\hline Yes $(\%)$ & 25 & 0 & 79.6 & 50 & $* * *<0.001$ & $* * *<0.001$ & $* * 0.001$ \\
\hline
\end{tabular}

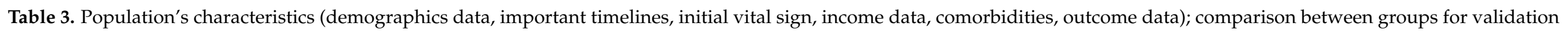
cohort. Parameters with a $p$-value $<0.05$ have significative differences between groups compared. ${ }^{*} p<0.05 ;{ }^{* *} p<0.01$; ${ }^{* * *} p<0.001$.

\begin{tabular}{|c|c|c|c|c|c|c|c|}
\hline & All $(n=140)$ & $\begin{array}{c}\text { SMU Group }^{\dagger} \\
\quad(n=87)\end{array}$ & $\begin{array}{c}\text { ICU Group }^{\dagger} \\
\quad(n=10)\end{array}$ & $\begin{array}{c}\text { SToI Group }^{\dagger} \\
\quad(n=43)\end{array}$ & $\begin{array}{l}\text { SMU vs. SToI } \\
\quad(p \text {-Value })\end{array}$ & $\begin{array}{c}\text { SMU vs. ICU } \\
(p \text {-Value })\end{array}$ & $\begin{array}{c}\text { SToI vs. ICU } \\
\text { ( } p \text {-Value) }\end{array}$ \\
\hline \multicolumn{8}{|l|}{ Demographics characteristics } \\
\hline Age. years. median [IQR] & $71[61-81]$ & $75[62-85]$ & $67[59-74]$ & 67 [59-72] & $* * 0.001$ & 0.112 & 0.641 \\
\hline Age $\geq 75$ years $(\%)$ & 39.3 & 52.9 & 20.0 & 16.3 & $* * *<0.001$ & $* * *<0.001$ & 1 \\
\hline Medically assisted nursing home & 7.9 & 11.5 & 10.0 & 0.0 & $* 0.03$ & $* 0.043$ & 0.189 \\
\hline Gender Male (\%) & 61.4 & 55.2 & 80 & 69.8 & 0.110 & 0.154 & 0.706 \\
\hline \multicolumn{8}{|l|}{ Timeline (day) } \\
\hline $\begin{array}{l}\text { Time between first symptoms and hospitalisation. median } \\
\text { [IQR] }\end{array}$ & $5[3-7]$ & 5 [3-7] & $7[2-13]$ & $5[3-7]$ & 0.931 & 0.177 & 0.231 \\
\hline Time between SMU and ICU. median [IQR] & & & & $4[2-6]$ & & & \\
\hline Time in SMU. median [IQR] & & $8[6-12]$ & & & & & \\
\hline Time in ICU. median [IQR] & & & $11[6-17]$ & $7[3-20]$ & & & \\
\hline \multicolumn{8}{|l|}{ Initial clinical characteristics } \\
\hline \multicolumn{8}{|l|}{ Body Mass Index $\left(\mathrm{kg} / \mathrm{m}^{2}\right)$} \\
\hline$<18.5(\%)$ & 0,0 & 0.0 & 0.0 & 0.0 & & & \\
\hline$\geq 30(\%)$ & 27.1 & 18.4 & 20.0 & 46.5 & ** 0.001 & ** 0.005 & 0.286 \\
\hline
\end{tabular}


Table 3. Cont.

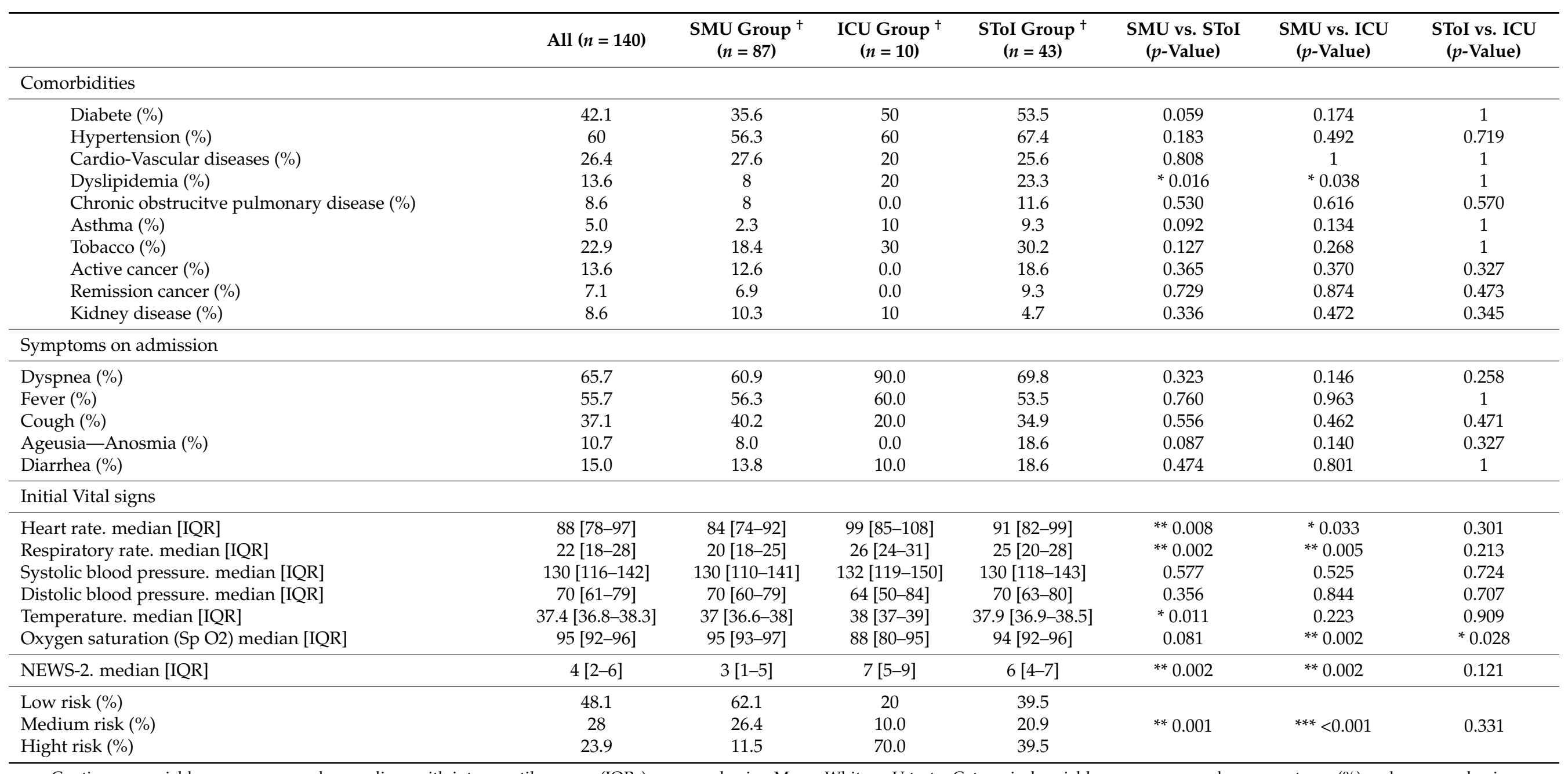

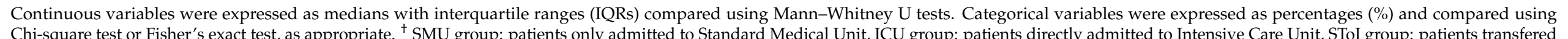
from Standard medical unit to Intensive care unit. 


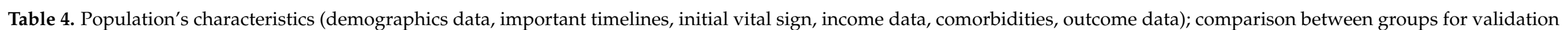

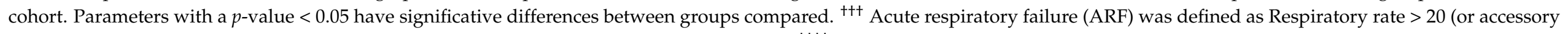

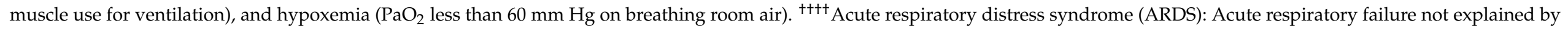

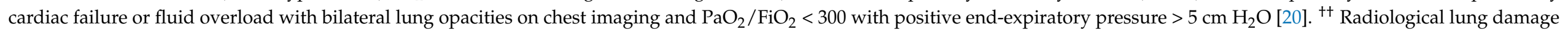

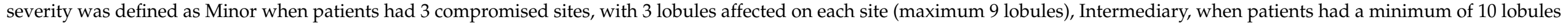

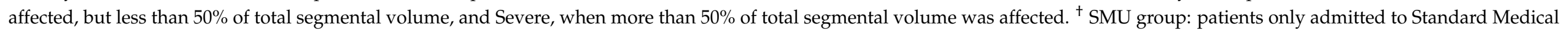

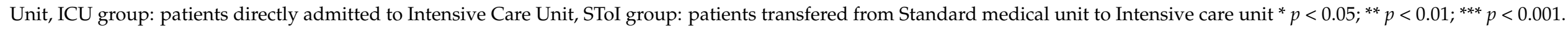

\begin{tabular}{|c|c|c|c|c|c|c|c|}
\hline & All $(n=140)$ & $\begin{array}{c}\text { SMU Group }^{\dagger} \\
\quad(n=87)\end{array}$ & $\begin{array}{l}\text { ICU Group }^{\dagger} \\
\quad(n=10)\end{array}$ & $\begin{array}{l}\text { SToI Group } \\
\quad(n=43)\end{array}$ & $\begin{array}{l}\text { SMU vs. SToI } \\
\text { (p-Value) }\end{array}$ & $\begin{array}{l}\text { SMU vs. ICU } \\
\text { (p-Value) }\end{array}$ & $\begin{array}{c}\text { SToI vs. ICU } \\
\text { ( } p \text {-Value) }\end{array}$ \\
\hline \multicolumn{8}{|l|}{ Initial $\mathrm{O} 2$ needed } \\
\hline Yes $(\%)$ & 34.3 & 32.2 & 30 & 39.5 & 0.407 & 0.714 & \multirow[t]{2}{*}{0.725} \\
\hline Volume. median [IQR] & $3[2-5]$ & $3[2-5]$ & NA & $3[2-4]$ & 0.885 & *0.019 & \\
\hline \multicolumn{8}{|l|}{ Computed tomography (CT) low dose COVID-19 } \\
\hline Yes $(\%)$ & 96.4 & 95.4 & 88.9 & 100 & 0.301 & 0.140 & 0.173 \\
\hline Absence $(\%)$ & 5.7 & 8.4 & 0.0 & 2.4 & \multirow{4}{*}{0.1} & \multirow{4}{*}{0.070} & \multirow{4}{*}{0.496} \\
\hline Minor $(\%)$ & 25.7 & 30.1 & 12.5 & 23.8 & & & \\
\hline Intermediary (\%) & 32.1 & 38.6 & 12.5 & 28.6 & & & \\
\hline Severe $(\%)$ & 31.4 & 22.9 & 75.0 & 45.2 & & & \\
\hline \multicolumn{8}{|l|}{ Outcomes } \\
\hline Pulmonary embolism (\%) & 2.9 & 1.1 & 10.0 & 4.7 & \multirow[t]{2}{*}{0.254} & 0.093 & \multirow[t]{2}{*}{0.473} \\
\hline Cerbebral strocke (\%) & 0 & 0 & 0.0 & 0.0 & & & \\
\hline Deep vein thrombosis (\%) & 0.7 & 0 & 0.0 & 2.3 & 0.331 & 0.379 & 1 \\
\hline Azithomycin $(\%)$ & 42.9 & 96.6 & 70.0 & 79.1 & ** 0.002 & $* * 0.001$ & 0.677 \\
\hline Hydroxychloroquine (\%) & 89.3 & 44.8 & 10.0 & 46.5 & 0.856 & 0.096 & 0.069 \\
\hline Acute repiratory failure ${ }^{++\dagger}(\%)$ & 50.7 & 23 & 90.0 & 97.7 & $* * *<0.001$ & $* * *<0.001$ & 1 \\
\hline Acute respiratory distress syndrome $[\mathrm{ARDS}]^{++++}(\%)$ & 40.7 & 9.2 & 90.0 & 93 & $* * *<0.001$ & $* * *<0.001$ & 1 \\
\hline Death $(\%)$ & 13 & 9.2 & 20.0 & 26.2 & *0.011 & *0.026 & 1 \\
\hline Maximum O2 help & & & & & & & \\
\hline
\end{tabular}


Table 4. Cont.

\begin{tabular}{|c|c|c|c|c|c|c|c|}
\hline & All $(n=140)$ & $\underset{(n=87)}{\text { SMU Group }^{+}}$ & $\begin{array}{c}\text { ICU Group }^{\dagger} \\
\quad(n=10)\end{array}$ & $\begin{array}{c}\text { SToI Group }^{+} \\
\quad(n=43)\end{array}$ & $\begin{array}{l}\text { SMU vs. SToI } \\
\text { (p-Value) }\end{array}$ & $\begin{array}{l}\text { SMU vs. ICU } \\
\text { (p-Value) }\end{array}$ & $\begin{array}{l}\text { SToI vs. ICU } \\
\text { ( } p \text {-Value) }\end{array}$ \\
\hline \multicolumn{8}{|l|}{ High-concentration mask } \\
\hline Yes $(\%)$ & 19.3 & 10.3 & & 41.9 & $* * 0.004$ & $* * 0.006$ & 0.345 \\
\hline O2 Volume (1 / min). median [IQR] & $40[28-50]$ & 15 [15-25] & NA & $45[35-50]$ & $* * *<0.001$ & & \\
\hline \multicolumn{8}{|l|}{ Oro-tracheal intubation } \\
\hline Yes $(\%)$ & 20.7 & 0 & 80.0 & 48.8 & $* * *<0.001$ & $* * *<0.001$ & 0.091 \\
\hline
\end{tabular}

Table 5. NEWS (National Early Warning Score) 2 scoring system calculation and interpretation.

\begin{tabular}{|c|c|c|c|c|c|c|c|}
\hline \multirow{2}{*}{ Physiological Parameter } & \multicolumn{7}{|c|}{ Score } \\
\hline & +3 & +2 & +1 & 0 & +1 & +2 & +3 \\
\hline Respiration rate (per min ute) & $\leq 8$ & & $9-11$ & $12-20$ & & $21-24$ & $\geq 25$ \\
\hline $\mathrm{SpO}_{2}$ scale $1(\%)^{*}$ & $\leq 91$ & $92-93$ & $94-95$ & $\geq 96$ & & & \\
\hline $\mathrm{SpO}_{2}$ scale $2(\%) *$ & $\leq 83$ & $84-85$ & $86-87$ & $\begin{aligned} & 88-92 \\
& \geq 93 \text { on air } \\
&\end{aligned}$ & $\begin{array}{c}\text { 93-94 } \\
\text { on oxygen }\end{array}$ & $\begin{array}{c}\text { 95-96 } \\
\text { on oxygen }\end{array}$ & $\begin{array}{c}\geq 97 \\
\text { on oxygen }\end{array}$ \\
\hline Air or oxygen? & & Oxygen & & Air & & & \\
\hline $\begin{array}{l}\text { Heart rate } \\
\text { (per minute) }\end{array}$ & $\leq 40$ & & $41-50$ & $51-90$ & $91-110$ & $111-130$ & $\geq 131$ \\
\hline Consciousness & & & & Alert & & & $\begin{array}{l}\text { New-onset confusion (or } \\
\text { disorientation/agitation) }\end{array}$ \\
\hline Temperature $\left({ }^{\circ} \mathrm{C}\right)$ & $\leq 35.0$ & & $35.1-36.0$ & $36.1-38.0$ & $38.1-39.0$ & $\geq 39.1$ & \\
\hline NEWS2 interpretation & & & & $\begin{array}{r}\text { Aggregate scol } \\
\text { Aggregate score } \\
\text { Aggregate score }=\end{array}$ & $\begin{array}{l}\text { 4: Low clinic } \\
\text { Medium clir } \\
\text { bove: High c. }\end{array}$ & sk & \\
\hline
\end{tabular}

* Oxygen saturation $\left(\mathrm{SpO}_{2}\right)$ Scale 1: $\mathrm{SpO}_{2}$ on room air or supplemental $\mathrm{O}_{2}$ if patient has no hypercapnic respiratory failure. SpO $\mathrm{S}_{2} \mathrm{Scale} 2$ : If patient has hypercapnic respiratory failure. 
This parameter is still significative in a multivariate analysis, including body mass index, respiratory rate, $\mathrm{SpO}_{2}$, neutrophil-lymphocyte ratio, C-reactive protein and lactate dehydrogenase comparing SMU vs. (ICU + SToI) groups (OR 231.2; 95\% CI: [8.1-,611.4]; $p=0.001]$ ) (Table 6). All multivariate analysis always used same parameters (age, body mass index, respiratory rate, $\mathrm{SpO}_{2}$, neutrophil-lymphocyte ratio, C-reactive protein and lactate dehydrogenase).

Table 6. Statistical multivariate analysis of PREDICT score parameters during the first two days of hospitalization in standard medical unit (SMU). The two highest severity groups: SToI (need transfer to intensive care unit (ICU)) are compared to the referential group (Standard Medical Unit (SMU)). Parameters with a $p$-value $<0.05$ have significative differences between groups compared.

\begin{tabular}{|c|c|c|c|c|c|c|c|}
\hline & & & & & Odd Ratio & $\begin{array}{c}\text { Confidence } \\
\text { Interval (95\%) }\end{array}$ & $p$-Value \\
\hline & \multicolumn{2}{|c|}{ Day 0} & \multicolumn{2}{|c|}{ Day 0} & \multicolumn{3}{|c|}{ Day 0} \\
\hline Admission parameters & \multicolumn{2}{|c|}{ SMU Group } & \multicolumn{2}{|c|}{ SToI + ICU Groups } & \multicolumn{3}{|c|}{ SMU vs. (SToI + ICU) } \\
\hline Age $<75$ years & \multicolumn{2}{|c|}{$50.8 \%$} & \multicolumn{2}{|c|}{$76 \%$} & 231.2 & {$[8.1 ; 6,611.4]$} & $* * 0.001$ \\
\hline Body Mass Index $\geq 30 \mathrm{~kg} / \mathrm{m}^{2}$ & \multicolumn{2}{|c|}{$22.9 \%$} & \multicolumn{2}{|c|}{$29.1 \%$} & 96.4 & {$[4.8 ; 1,928.1]$} & $* * 0.003$ \\
\hline $\begin{array}{c}\text { Respiratory rate } \\
\geq 23 \text { breaths/min }\end{array}$ & \multicolumn{2}{|c|}{$40 \%$} & \multicolumn{2}{|c|}{$64.1 \%$} & 348.7 & {$[10 . ; 11,567.9]$} & $* * 0.001$ \\
\hline $\begin{array}{c}\text { Oxygen saturation } \leq 95 \% \\
\text { (room air) }\end{array}$ & \multicolumn{2}{|c|}{$46.3 \%$} & \multicolumn{2}{|c|}{$64.1 \%$} & 244.6 & {$[9.2 ; 6,490.1]$} & $* * 0.001$ \\
\hline \multirow[t]{2}{*}{$\begin{array}{l}\text { Neutrophil-to-Lymphocyte } \\
\text { Ratio } \geq 4\end{array}$} & \multicolumn{2}{|c|}{$51.8 \%$} & \multicolumn{2}{|c|}{$80.6 \%$} & 36.9 & {$[1.1 ; 1,258.9]$} & $* 0.045$ \\
\hline & \multicolumn{2}{|c|}{ Day 1} & \multicolumn{2}{|c|}{ Day 2} & \multicolumn{3}{|c|}{ Day 1 and 2} \\
\hline Following parameters & SMU Group & SToI Group & SMU Group & SToI Group & \multicolumn{3}{|c|}{ SMU vs. SToI } \\
\hline $\begin{array}{c}\text { Neutrophil-lymphocyte } \\
\text { Ratio } \geq 6\end{array}$ & $32.4 \%$ & $41.7 \%$ & $29.7 \%$ & $60 \%$ & 61.9 & {$[1.7 ; 2,192.3]$} & $* 0.023$ \\
\hline C- Reactive protein $\geq 53 \mathrm{mg} / \mathrm{L}$ & $61.3 \%$ & $80 \%$ & $65.8 \%$ & $85.2 \%$ & 2987.5 & {$[10.7 ; 836,567.9]$} & $* * 0.005$ \\
\hline $\begin{array}{l}\text { Lactate Dehydrogenase } \\
\geq 450 \mathrm{UI} / \mathrm{L}\end{array}$ & $15.5 \%$ & $35.5 \%$ & $6.3 \%$ & $64 \%$ & 60.6 & {$[3.1 ; 1,174.4]$} & $* * 0.007$ \\
\hline
\end{tabular}

Moreover, body mass index superior or equal to 30 has already been observed [21] as key comorbid factor in the intensive care units. In this study, the percentage of subjects with body mass index superior or equal to 30 was $22.9 \%, 22.1 \%$ and $49 \%$ in the SMU, SToI and ICU groups, respectively, representing a significative difference. Multivariate analysis for this criterion also showed statistical significance, with an odds ratio 96.4 (95\% CI: [4.8-1928.1]; $p=0.003)$, patients with a body mass index superior to 30 had greater risk of needing intensive care unit treatment (Table 6).

However, only parameters identified as independent risk factors through multivariate regression analysis were used to build the score: age, body mass index, respiratory rate, and $\mathrm{SpO}_{2}$ (Tables 1 and 2). The same analysis and results are presented in Tables 3 and 4 for the validation cohort.

\subsection{Patient Vital Signs}

Notable differences appeared after analyzing differences between groups on easily measurable vital signs (respiratory rate, temperature, $\mathrm{SpO}_{2}$ ) (Tables 1 and 3).

Vital parameters for SMU, SToI and ICU groups were: respiratory rate (Median: 22, IQR: 18-26), (Median: 24, IQR: 20-30), and (Median: 30, IQR: 25-35), respectively; body temperature (Median: 37.1, IQR: 36.7-38), (Median: 37.9, IQR: 37-38.5), and (Median: 38.1, IQR: 37.1-38.8), respectively; $\mathrm{SpO}_{2}$ (Median: 96, IQR: 93-97), (Median: 95, IQR: 93-96), (Median: 94, IQR: 89-95), respectively. 
Multivariate analysis for those criteria showed a statistical significance (OR 348.6 (IC 95\%: 10.5-11,567.9; $p=0.001$ ) and OR 244.6 (IC 95\%: 9.2-6,490.1; $p=0.005$ ), respectively, for respiratory rate and $\mathrm{SpO}_{2}$ ) (Table 6).

\subsection{Patient Biological Parameters}

Regarding patient vital signs, notable differences were observed in general kinetic biological parameters between groups (C-reactive protein, neutrophil-lymphocyte ratio, Albuminemia, lactate dehydrogenase, Fibrinogen) (Figure 2).
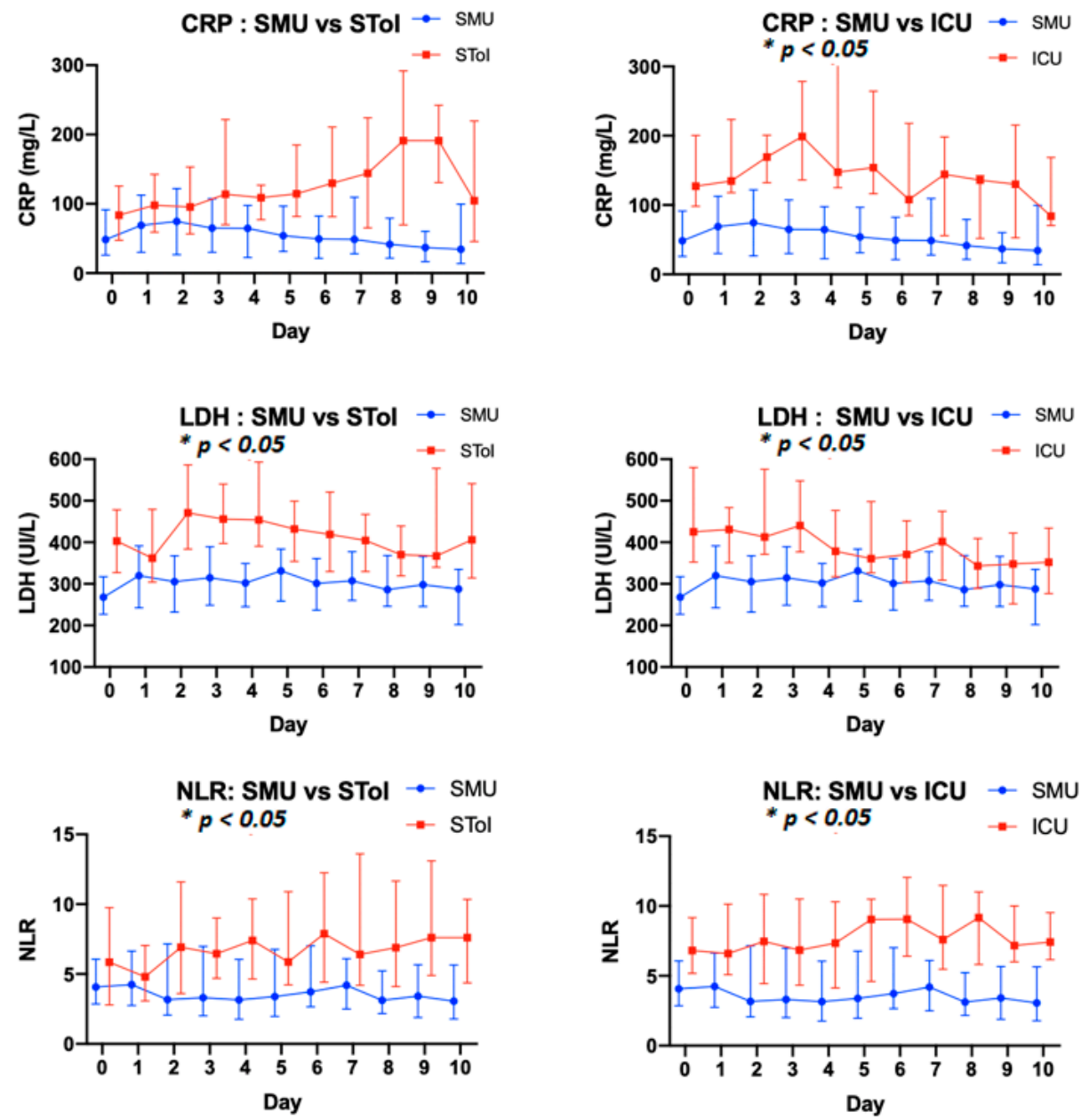

Figure 2. Kinetic following of biological parameters in training cohort (media and interquartile). Left column: Standard Medical Unit Patients vs. Standard to Intensive Care Patients groups. Right column: Standard Medical Unit Patients vs. Intensive Care Units Patients. NLR: neutrophil-lymphocyte ratio, CRP: C-reactive protein, $\mathrm{LDH}$ : lactate dehydrogenase. Data were expressed as mean and range. Statistical analysis was performed to compare the kinetics of biological parameters over time (Day 0 to Day 10) between groups of patients (see Statistical analysis). ${ }^{*} p<0.05$ mean that there was a significant difference in the behavior of parameters.

Linear mixed models were performed for biological parameters, showing significant differences between the SMU, SToI and ICU groups during the two first days of hospitalization (Table 6), for PREDICT score training. The two-day timeframe was chosen because it represents the first quartile of time in which the standard medical unit to intensive care unit switch occurred in the SToI group. Furthermore, this follow-up period had to be long enough to provide enough time for physicians to react to and manage their patients and resources. 
Results comparing SMU group versus SToI groups report Odds Ratios (OR) for C-Reactive Protein, Neutrophil-Lymphocyte ratio, and Lactate dehydrogenase of 2987.5 (95\% CI: 10.7-836,6, p: 0.005), 61.9 (95\% CI:1.7-2192.3, p: 0.023), and 60.6 (95\% CI:3.1-1,17, $p$ : 0.007), respectively, showing an increase in those parameters during the two first days of hospitalization.

\subsection{Clinical-Biological Score for Predicting Intensive Care Risk}

Considering all previous results, the most pertinent parameters were chosen to develop a score that is able to help physicians anticipate their patients' deterioration and prepare for their transfer to intensive care unit, thus improving resource management.

This score can be calculated at admission (day 0), and then at day one and day two of hospitalization. It can deal with missing data for kinetic biological follow-up. It has an all-or-none approach for each criterion. For example, if a patient has a body mass index superior to 30, the score user must add nine points; if the patient has a body mass index lower than 30, no points are added.

Three optimal thresholds were determined by maximum Youden index calculated on all points of the receiver operating characteristic curve. ROC curves are represented in Figure 3; they both have area under curve superior to 0.7 and they are all statistically significant, with a $p$-value inferior to 0.0001. Population division based on the PREDICT score is shown in Figure 4.

ROC curve: Admission PREDICT Score

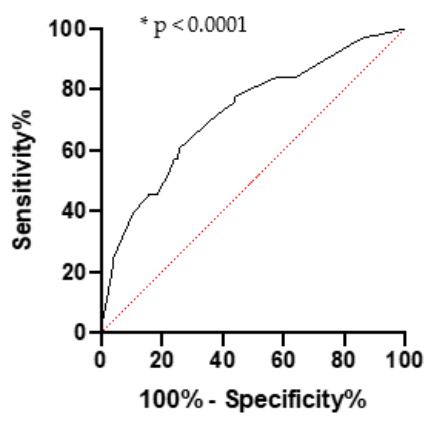

ROC curve: Day 2 PREDICT Score

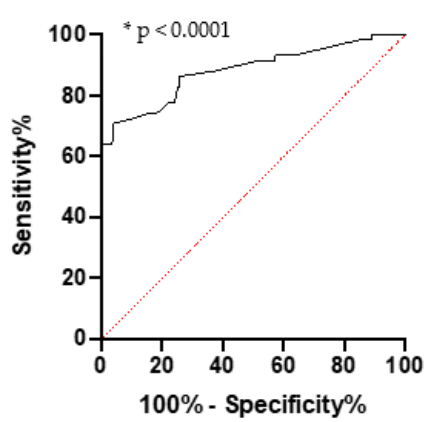

ROC curve: Day 1 PREDICT Score
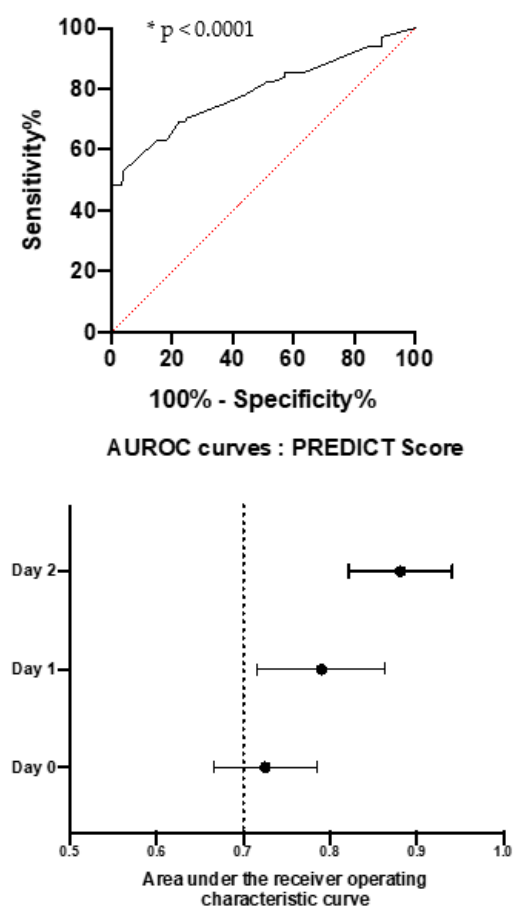

Figure 3. Receiver Operating Characteristic (ROC) curves for PREDICT score on admission in Intensive Care Unit; Day 0, day 1, and day 2 of hospitalization, and area under ROC curve repartition. $* p<0.05$.

If a patient has a PREDICT score superior to the cut-off (Day 0: 25, Day 1: 34, Day 2: 35), no matter the day of calculation, he has an important risk of needing intensive care during hospitalization (Table 7). 
Admission PREDICT Score distribution

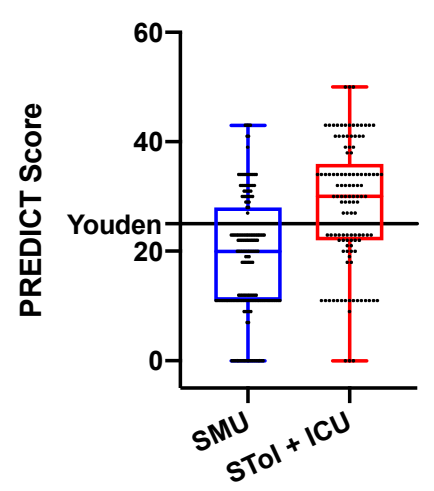

Day 1 PREDICT Score distribution

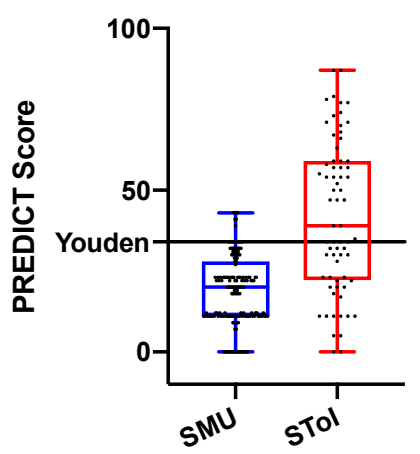

Day 2 PREDICT Score distribution

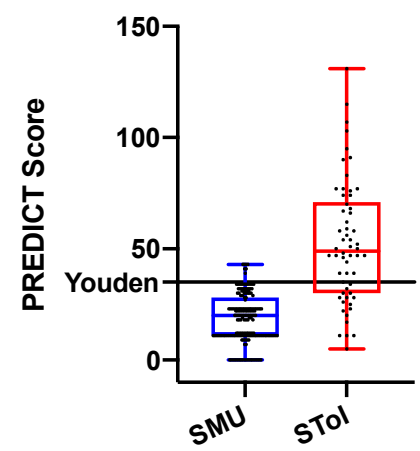

Figure 4. PREDICT score population construction repartition during the first two days of hospitalization, with maximum Youden index value (Cut-off). SMU: standard medical unit. SToi: need intensive care unit (ICU).

Table 7. PREDICT score calculation table for the transfer to intensive care unit (ICU). Calculate Day 0 score by a simple sum. Day 1 score is the sum of Day 0 score plus day 1 biological potential point plus following adjustment 1 or 2 . Finally, Day 2 score is the sum of day 1 score plus day 2 biological potential point plus following adjustment 1 or 2 .

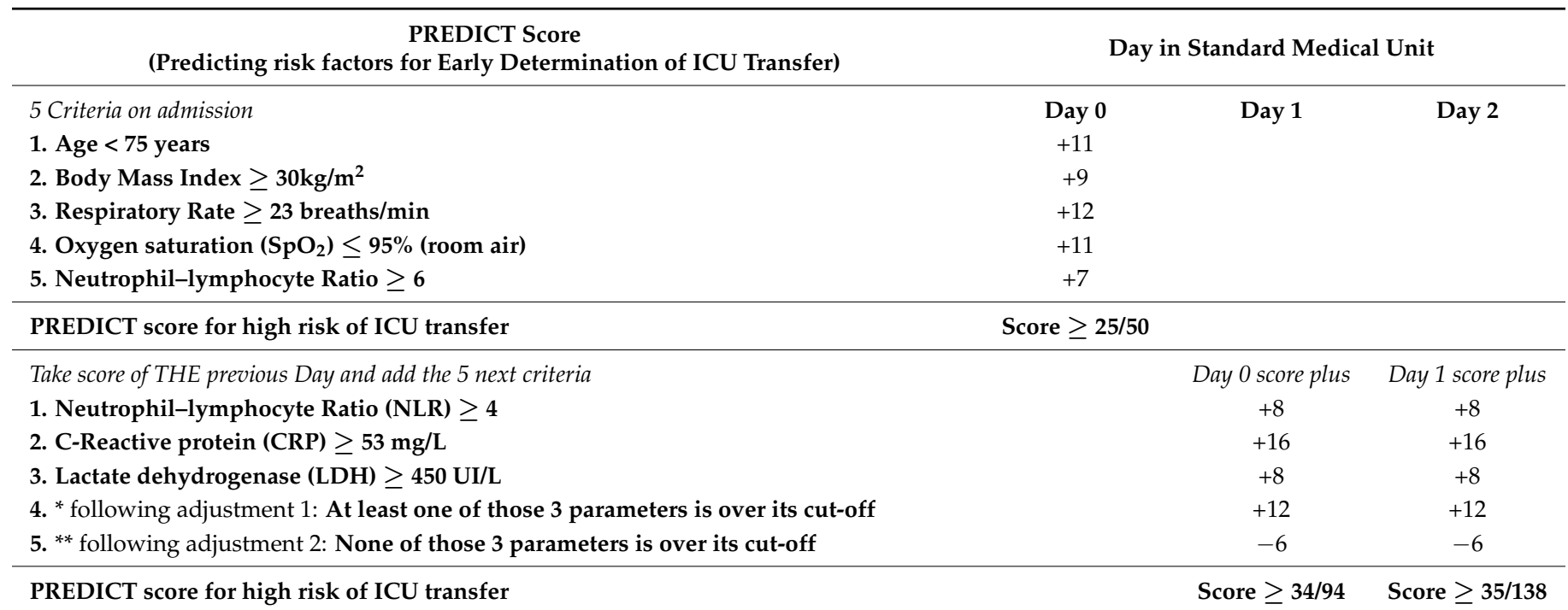

* Following adjustment 1: if a patient has almost one biological parameters (neutrophil-lymphocyte ratio, C-reactive protein, lactate dehydrogenase) over the threshold, add 12 points. ${ }^{* *}$ Following adjustment 2: if a patient has no biological parameters (neutrophillymphocyte ratio, C-reactive protein, lactate dehydrogenase) over the threshold, subtract 6 points.

\section{Discussion}

The major point that emerges from the present study is that the PREDICT score is useful to screen the COVID-19 hospitalized patients to locate those who need to be transferred to an intensive care unit. The population of this study is comparable to previous studies for baseline parameters [22,23] (gender, age, etc.). Furthermore, comorbidities like hypertension (defined using recent guidelines [24]), diabetes, and dyslipidemia showed similar prevalence rates, as reported by previous publications $[25,26]$.

For example, the prevalence of obesity found in the SMU, SToI and ICU groups was $22.9 \%, 22.1 \%$, and $49 \%$, respectively. To simplify, we divided body mass index into only four categories (underweight, normal weight, overweight and obesity), without separating by obesity levels. Body mass index $\geq 30$ was found to be a strong positive independent risk factor between our baseline population (SMU group) and ICU risk population. This very important proportion of patients with a body mass index $\geq 30$ in need of ICU care has already been reported [21]. However, few significant differences between groups 
were found regarding other comorbidities, in contrast to what has been reported by other studies [27]. We believe that this is because the population included in our study already has a degree of disease severity, as it is composed of patients with a form of COVID-19 severe enough to warrant hospitalization. Thus, the comparison in our study is not with the general population, as has been in other studies, but with a population of hospitalized patients, whose baseline characteristics probably involve a higher degree of comorbidity.

Furthermore, a second crucial threshold was highlighted in this study. Age $<75$ years, ages inferior to 75 years, were more likely to be admitted to intensive care unit by a statistically significant Odds Ratio (OR) after comparing the SMU group to the SToI and ICU groups. Such an Odds Ratio was previously found in the French national database [4] (Table 2, which allowed us to calculate this Odds Ratio to 5.6 (95\% CI: [5.2-9.9]; $p<0.0001$ ). This observation is surprising; however, two explanations could be proposed. First of all, the attack rate of SARS CoV-2 created a patient flow that surpassed our health care system's capacity, imposing the need for a war-like medical triage system, in which the limited number of beds in intensive care units were assigned to patients that had the most chance of survival. Further, frail patients were admitted in a serious state, perhaps because of the hypoxic happiness phenomenon [28]

The main strength of our study is the kinetic follow-up of biological parameters. The study included common biological parameters, as we aimed to build a very userfriendly score. Further, it showed trends in accordance with previous analysis reported in the literature [29]. The comparison of kinetic follow-up during the two first days of hospitalization, with significative differences in C-reactive protein, lactate dehydrogenase and neutrophil-lymphocyte ratio, allowed us to apply our score during those first days. In clinical practice, this would allow physicians a comfortable time in which to evaluate the patient's clinical course and react if necessary.

In Predict score at Day 0, the proportion of patients in the SMU groups who simultaneously reached the next three criteria: Age $<75$ years and $\mathrm{SpO}_{2} \leq 95 \%$ and respiratory rate $\geq 23$ breaths $/ \mathrm{min}$ was $9.7 \%$ in the training cohort and $9.1 \%$ in the validation cohort.

In our hospital, patients needing only high-flow oxygen treatment are managed in standard medical monitoring units. Admission to the ICU is indicated if desaturation occurs despite maximal high-flow oxygen therapy or another organ failure appears (cardiac, neurologic, hepatic or renal).

Recent studies highlight the importance of biomarkers like D-Dimer, anticardiolipin IgG autoantibody, C-reactive protein, and interleukin-6 in the prediction of COVID-19 patients' clinical decline [30,31]. Data regarding D-Dimer and albuminemia levels in our population show a trend towards higher D-dimer levels and lower albumin levels in both the STol and ICU groups (Figure 5). However, because our study was initiated at a time of crisis in France, there is a lack of data, which only allowed us to observe trends and prevented us from demonstrating statistical significance; this is a limitation to our study. Further, we were unable to demonstrate an association between the level of hypoalbuminemia during COVID-19 infection and risk of intensive care because of the confounding impact of dilution, despite previous studies showing its importance [32,33].

Moreover, a study proposing NEWS 2 as a tool for identifying patients at risk of requiring intensive care has been previously published [12], and we have compared its characteristics to those of our PREDICT score using a threshold of 5 for NEWS 2, as has been proposed (Tables 8-11). NEWS 2 score is composed with the following variables: respiratory rate, oxygen saturation, need for supplemental oxygen, body temperature, blood pressure, heart rate and level of consciousness. In Anna Gidari publication [12], a threshold of 5 points in NEWS 2 is recommended for monitoring patients. The PREDICT score achieved a good sensitivity and a very good negative predictive value, which increased with each day it was performed, during the time allowed by the score (admission, day one and finally day two of hospitalization). A key point that emerges in both the training cohort and the validation cohort is the ability of the PREDICT score to correctly predict the need for transfer to intensive care of a patient already hospitalized for the management of COVID-19. 
In the SToI group of the training and validation cohorts, in patients in whom the PREDICT score was calculated only twice (those who went into intensive care after exactly $24 \mathrm{~h}$ of hospitalization had their PREDICT score calculated twice: at admission and day 1); at least one calculation was positive in $100 \%$ and $83.3 \%$, respectively. Further, patients who needed transfer to intensive care unit after $48 \mathrm{~h}$ of hospitalization had a positive PREDICT score at least one time on admission, at day one or day two of hospitalization, with a $100 \%$ identification rate in both the training cohort and validation cohort.
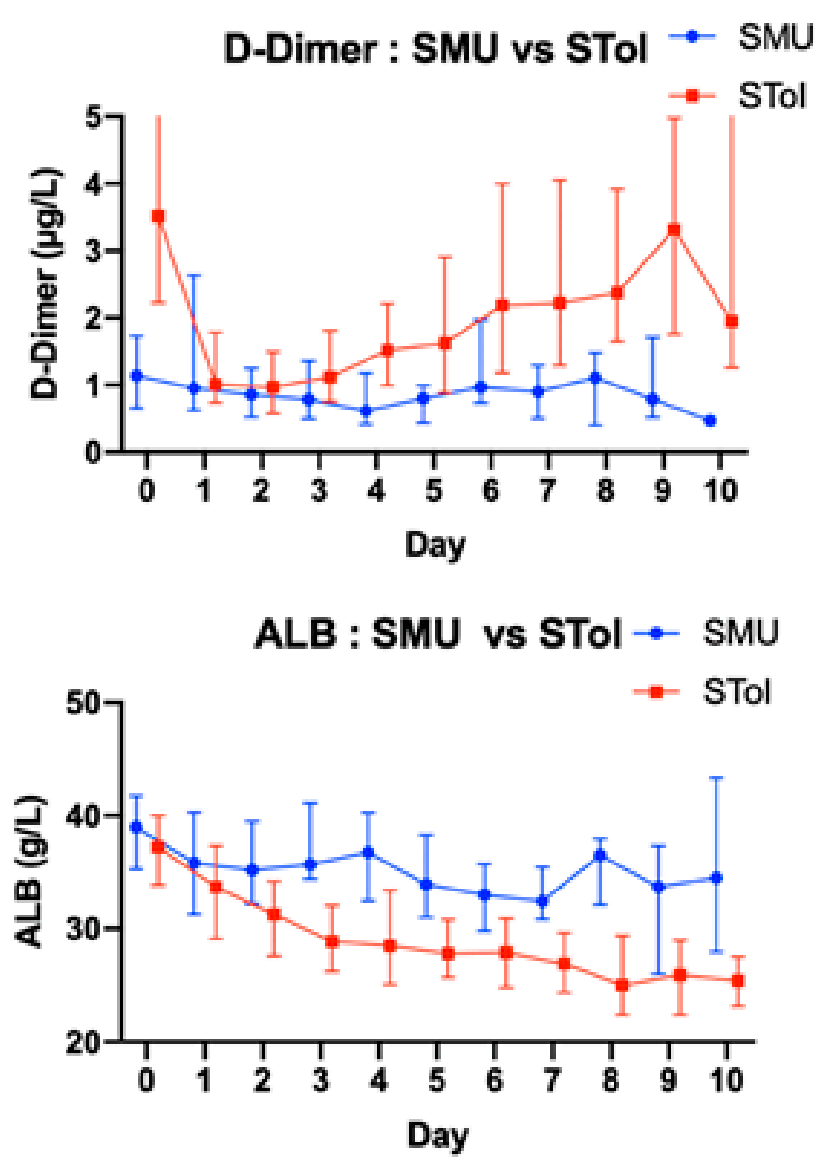
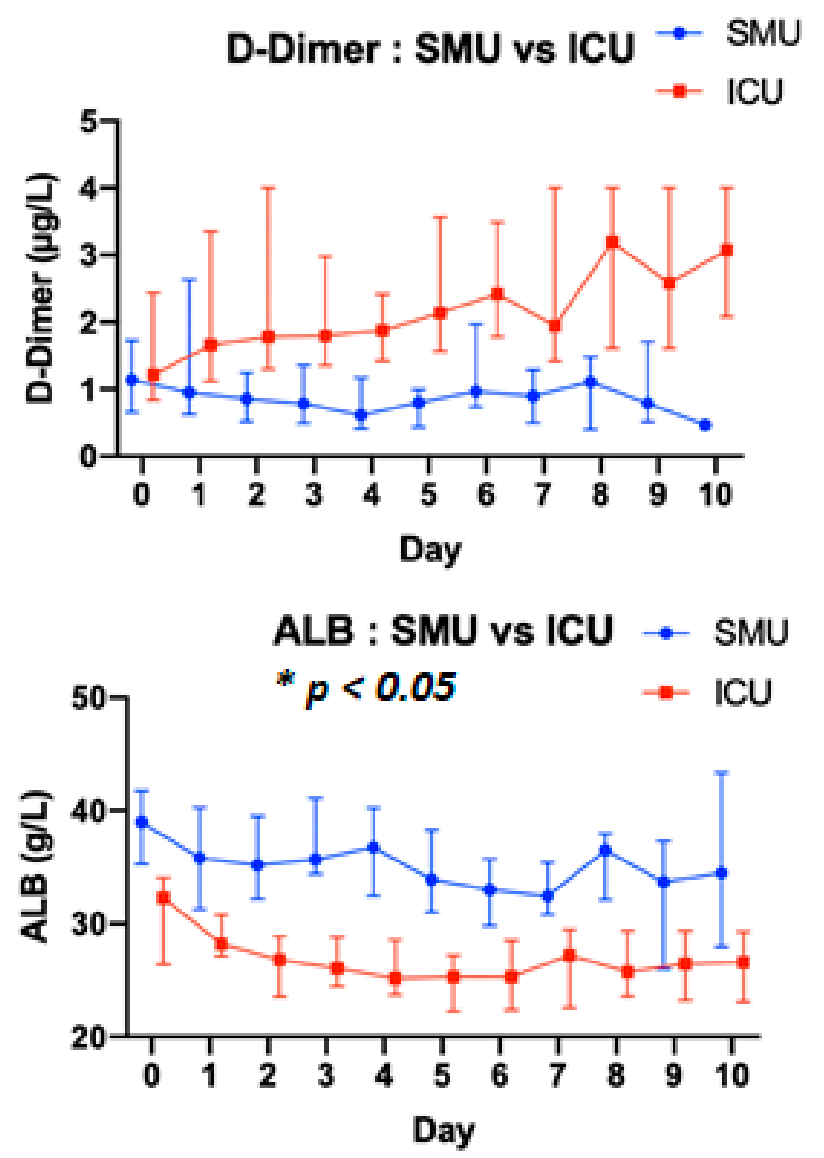

Figure 5. Kinetic follow-up of biological parameters. Left column: Standard Medical Units Patients vs. Standard to Intensive Care Patients. Right column: Standard Medical Units Patients vs. Intensive Care Units Patients. ALB: albuminemia.

Table 8. PREDICT and NEWS2 score characteristic comparison for training cohort. Se: sensitivity; Sp: specificity; PPV: Positive Predictive Value; NPV: Negative Predictive Value.

\begin{tabular}{cccccc}
\hline \multicolumn{5}{c}{ Training Cohort } \\
\hline NEWS & D0 & Se & $71.4 \%$ & Sp & $61.0 \%$ \\
Threshold & 5 & PPV & $54.7 \%$ & NPV & $76.4 \%$ \\
\hline PREDICT & D0 & Se & $60.7 \%$ & Sp & $74.3 \%$ \\
Youden & 25 & PPV & $61.2 \%$ & NPV & $73.9 \%$ \\
\hline \multirow{2}{*}{ Youden } & D1 & Se & $58.8 \%$ & Sp & $65.7 \%$ \\
\hline \multirow{2}{*}{ Youden } & 34 & PPV & $40.0 \%$ & NPV & $80.4 \%$ \\
\hline
\end{tabular}


Table 9. PREDICT and NEWS2 score characteristic comparison for validation cohort. Se: sensitivity; Sp: specificity; PPV: Positive Predictive Value; NPV: Negative Predictive Value.

\begin{tabular}{cccccc}
\hline \multicolumn{5}{c}{ Validation Cohort } \\
\hline NEWS & D0 & Se & $79.1 \%$ & Sp & $62.1 \%$ \\
Threshold & 5 & PPV & $50.7 \%$ & NPV & $85.7 \%$ \\
\hline PREDICT & D0 & Se & $54.7 \%$ & Sp & $80.5 \%$ \\
Youden & 25 & PPV & $63.0 \%$ & NPV & $74.5 \%$ \\
\hline Youden & D1 & Se & $51.2 \%$ & Sp & $59.8 \%$ \\
\hline Youden & 34 & PPV & $38.6 \%$ & NPV & $71.2 \%$ \\
\hline
\end{tabular}

Table 10. Percentages of patients that have at least one positive occurrence for PREDICT score before their switch day (calculated at day 0 for ICU group, day 0 and day 1 for patients who switch after 1 day of hospitalization in SToI group, day 0 and day 1 and day 2 for patients who switch after at least 2 days of hospitalization in SToI group). Line SMU represent the percentage of patient with a positive score at least one time (day 0 and day 1 and day 2) in SMU group.

\begin{tabular}{ccccccc}
\hline \multicolumn{7}{c}{ Training Cohort } \\
\hline Groups & \multicolumn{5}{c}{$\begin{array}{c}\text { PREDICT (\% of Patients with at Least 1 } \\
\text { Occurrence Positive before Switch) }\end{array}$} & $\begin{array}{c}\text { NEWS } \\
\text { (Admission) }\end{array}$ \\
\hline Day of switch to ICU & Day 0 & Day 1 & Day 2 & $>$ Day 2 & Total & Total \\
\hline SToI & & $100.0 \%$ & $100.0 \%$ & $77.1 \%$ & $83.8 \%$ & $56.9 \%$ \\
ICU & $77.6 \%$ & & & & $77.6 \%$ & $95.0 \%$ \\
SMU & & & & & $56.0 \%$ & $35.4 \%$ \\
\hline
\end{tabular}

Table 11. Percentages of patients that have at least one positive occurrence for PREDICT score before their switch day (calculated at day 0 for ICU group, day 0 and day 1 for patients who switch after 1 day of hospitalization in SToI group, day 0 and day 1 and day 2 for patients who switch after at least 2 days of hospitalization in SToI group). Line SMU represent the percentage of patient with a positive score at least one time (day 0 and day 1 and day 2) in SMU group.

\begin{tabular}{ccccccc}
\hline \multicolumn{7}{c}{ Validation Cohort } \\
\hline Groups & \multicolumn{4}{c}{$\begin{array}{c}\text { PREDICT (\% of Patients with at Least 1 } \\
\text { Occurrence Positive before Switch) }\end{array}$} & $\begin{array}{c}\text { NEWS } \\
\text { (Admission) }\end{array}$ \\
\hline Day of switch to ICU & Day 0 & Day 1 & Day 2 & $>$ Day 2 & Total & Total \\
\hline SToI & & $83.3 \%$ & $100.0 \%$ & $81.3 \%$ & $86.0 \%$ & $60.5 \%$ \\
ICU & $50.0 \%$ & & & & $50.0 \%$ & $80.0 \%$ \\
SMU & & & & & $52.9 \%$ & $37.9 \%$ \\
\hline
\end{tabular}

Moreover, in patients from the SToI groups of the training and validation cohorts, regardless of the day they transferred from SMU into ICU, PREDICT score was positive at least once out of the two or three possible attempts (admission and/or day 1 in patients who switch after $24 \mathrm{~h}$, and admission and/or day 1 and/or day 2 for those who switch after at least 2 days of hospitalization in SMU) in $83.8 \%$ and $86 \%$, respectively. Contrastingly, NEWS2 correctly sorted only $56.9 \%$ of patients in the training cohort and $60.5 \%$ of patients in the validation population. However, the PREDICT score has limitations, as it correctly sorts only $44 \%$ and $47.1 \%$ of SMU patients in the training and validation cohorts, respectively.

Recent publications show strong works, multivariate analysis, multicentric analysis, but with different approaches to the PREDICT score, without considering biological parameters, which can precede clinical signs [34] or needing a computer to be calculated, which 
is clearly powerful but less easy to use [35]. Even if the approaches are different, the goal is the same: saving lives. The PREDICT score, as any other scoring system, is a tool; it could be used in parallel with other tools because it provides another point of view.

\section{Conclusions}

The PREDICT score uses simple parameters, is easy to use, and manually calculable. This study shows the potential of this score to anticipate the risk of intensive care necessity for COVID-19 patients hospitalized in standard medical units. However, it is a tool that must be employed by medical professionals in combination with their clinical analysis of the patient's situation. The PREDICT score is powerful in identifying patients who require transfer from SMU to ICU, but less able to identify patients who need to be admitted to ICU in few hours; in such cases, the clinical sense of physicians is clearly dominant, and other tools, such as lactate values, could be employed. Moreover, the PREDICT Score classifies over $50 \%$ of patients hospitalized in SMU who will never need ICU care as in a risk category. Despite its imperfections, the PREDICT score correctly identifies patients who are at risk of needing intensive care.

Supplementary Materials: The following are available online at https:/ /www.mdpi.com/article/10 .3390 /biomedicines $9050566 /$ s1, Table S1. NEWS (National Early Warning Score) 2 scoring system calculation and interpretation.

Author Contributions: Conceptualization: M.G., J.F., R.G. Data curation: S.F., M.G. Formal analysis: S.F. Investigation: M.G., P.S., M.D. (Marine Duranjou), M.D. (Maelle Dambo), W.A. Methodology: M.G., J.F., R.G., S.F. Software: S.F. Supervision: J.F., R.G. Validation: M.G., J.F., R.G., P.M., N.B., M.M. Writing—original draft: M.G., J.F., R.G., P.M., N.B., S.F., M.M. Writing—review and editing: M.G., J.F., R.G., P.M., N.B., S.F., M.M. All authors have read and agreed to the published version of the manuscript.

Funding: This study was supported by Aix-Marseille University and Assistance Publique des Hôpitaux de Marseille. The authors received no specific funding for this work.

Institutional Review Board Statement: The study was conducted according to the guidelines of the Declaration of Helsinki, and approved by the ethics committee of Aix-Marseille University and Assistance Publique des Hôpitaux de Marseille.

Data Availability Statement: Data can be provided on C2VN (Aix Marseille University data base).

Acknowledgments: We thank all the members of our laboratories and departments for their continuous support.

Conflicts of Interest: The authors declare no conflict of interest. The funders had no role in study design, data collection and analysis, decision to publish, or preparation of the manuscript.

$\begin{array}{ll}\text { Abbreviations } & \\ \text { ICU } & \text { Intensive Care Unit } \\ \text { SMU } & \text { Standard Medical Unit } \\ \text { STol } & \text { Standard to Intensive Care } \\ \text { PREDICT score } & \text { Predicting Risk factors for Early Determination of ICU Transfer } \\ & \text { Assistance Publique des Hôpitaux de Marseille (Public Assistance } \\ \text { AP-HM } & \text { Hospital of Marseille) } \\ \text { Na } & \text { natremia } \\ \text { CRP } & \text { C-reactive protein } \\ \text { FRT } & \text { ferritinemia } \\ \text { LDH } & \text { lactate dehydrogenase } \\ \text { CREAT } & \text { creatinine } \\ \text { BILI } & \text { total bilirubin } \\ \text { ASAT } & \text { aspartate aminotransferase } \\ \text { ALAT } & \text { alanine aminotransferase } \\ \text { LY } & \text { lymphocyte count } \\ \text { NEU } & \text { neutrophils cells count }\end{array}$




$\begin{array}{ll}\text { NLR } & \text { neutrophil-lymphocyte ratio } \\ \text { WHO } & \text { World Health Organization } \\ \text { RT-PCR } & \text { real time polymerase chain reaction } \\ \mathrm{SpO}_{2} & \text { arterial oxygen saturation } \\ \text { ROC } & \text { receiver operating characteristic } \\ \mathrm{RR} & \text { respiratory rate } \\ \mathrm{T}^{\circ} \mathrm{C} & \text { temperature } \\ \text { IQR } & \text { interquartile range }\end{array}$

\section{References}

1. Huang, C.; Wang, Y.; Li, X.; Ren, L.; Zhao, J.; Hu, Y.; Zhang, L.; Fan, G.; Xu, J.; Gu, X.; et al. Clinical features of patients infected with 2019 novel coronavirus in Wuhan, China. Lancet 2020, 395, 497-506. [CrossRef]

2. World Health Organization. Novel Coronavirus (2019-nCoV): Situation Report 3. 2020. Available online: https://apps.who.int/ iris/handle/10665/330762 (accessed on 7 June 2020).

3. Ji, Y.; Ma, Z.; Peppelenbosch, M.P.; Pan, Q. Potential association between COVID-19 mortality and health-care resource availa-bility. Lancet Glob. Health 2020, 8, 480. [CrossRef]

4. Santé Publique France. COVID-19: Point Epidémiologique du 30 Avril 2020. Available online: https://maladies-et-traumatismes / maladies-et-infections-respiratoires / infection-a-coronavirus/documents/bulletin-national/covid-19-point-epidemiologiquedu-30-avril-2020 (accessed on 22 May 2020).

5. Semaine du 30 Mars 2020—CRES Paca. Available online: http:/ /www.cres-paca.org/a/885/semaine-du-30-mars-2020/ (accessed on 25 May 2020).

6. Plebani, M.; Laposata, M.; Lippi, G. A manifesto for the future of laboratory medicine professionals. Clin. Chim. Acta 2019, 489, 49-52. [CrossRef] [PubMed]

7. Lippi, G.; Plebani, M. The novel coronavirus (2019-nCoV) outbreak: Think the unthinkable and be prepared to face the chal-lenge. Diagnosis 2020, 7, 79-81. [CrossRef] [PubMed]

8. Lippi, G.; Plebani, M. A modern and pragmatic definition of Laboratory Medicine. Clin. Chem. Lab. Med. CCLM 2020. [CrossRef]

9. Gao, Y.; Li, T.; Han, M.; Li, X.; Wu, D.; Xu, Y.; Zhu, Y.; Liu, Y.; Wang, X.; Wang, L. Diagnostic utility of clinical laboratory data determinations for patients with the severe COVID-19. J. Med. Virol. 2020, 92, 791-796. [CrossRef]

10. Liang, W.; Liang, H.; Ou, L.; Chen, B.; Chen, A.; Li, C.; Li, Y.; Guan, W.; Sang, L.; Lu, J.; et al. Development and validation of a clinical risk score to predict the occurrence of critical illness in hospitalized patients with COVID-19. JAMA Intern. Med. 2020, 180, 1081-1089. [CrossRef]

11. Allenbach, Y.; Saadoun, D.; Maalouf, G.; Vieira, M.; Hellio, A.; Boddaert, J.; Gros, H.; Salem, J.E.; Rigon, M.R.; Menyssa, C.; et al. Development of a multivariate prediction model of intensive care unit transfer or death: A French prospective cohort study of hospitalized COVID-19 patients. PLoS ONE 2020, 15. [CrossRef]

12. Gidari, A.; Socio, G.V.D.; Sabbatini, S.; Francisci, D. Predictive value of National Early Warning Score 2 (NEWS2) for intensive care unit admission in patients with SARS-CoV-2 infection. Infect. Dis. 2020, 52, 698-704. [CrossRef]

13. World Health Organization. Laboratory Testing for 2019 Novel Coronavirus (2019-nCoV) in Suspected Human Cases. Available online: https: / www.who.int/publications-detail/laboratory-testing-for-2019-novel-coronavirus-in-suspected-humancases-20200117 (accessed on 27 May 2020).

14. Niederman, M.S.; Mandell, L.A.; Anzueto, A.; Bass, J.B.; Broughton, W.A.; Campbell, G.D.; Dean, N.; File, T.; Fine, M.J.; Gross, P.A.; et al. Guidelines for the management of adults with community-acquired pneu-monia. Am. J. Respir. Crit. Care Med. 2001, 163, 1730-1754. [CrossRef]

15. Metlay, J.P.; Waterer, G.W.; Long, A.C.; Anzueto, A.; Brozek, J.; Crothers, K.; Cooley, L.A.; Dean, N.C.; Fine, M.J.; Flanders, S.A.; et al. Diagnosis and treatment of adults with community-acquired pneumonia. An official clinical practice guideline of the american thoracic society and infectious diseases society of America. Am. J. Respir. Crit. Care Med. 2019, 200, 45-67. [CrossRef]

16. Lui, G. Viral dynamics of SARS-CoV-2 across a spectrum of disease severity in COVID-19. J. Infect. 2020, 1, 1-11. [CrossRef] [PubMed]

17. RCP London. National Early Warning Score (NEWS) 2. Available online: https://www.rcplondon.ac.uk/projects/outputs/ national-early-warning-score-news-2 (accessed on 27 May 2020).

18. Martinez, L.; Cheng, W.; Wang, X.; Ling, F.; Mu, L.; Li, C.; Huo, X.; Ebell, M.H.; Huang, H.; Zhu, L.; et al. A risk classification model to predict mortality among laboratory-confirmed avian influenza a H7N9 patients: A population-based observational cohort study. J. Infect. Dis. 2019, 220, 1780-1789. [CrossRef] [PubMed]

19. Redfern, O.C.; Smith, G.B.; Prytherch, D.R.; Meredith, P.; Inada-Kim, M.; Schmidt, P.E. A comparison of the quick sequential (sep-sis-related) organ failure assessment score and the national early warning score in non-ICU patients with/without infec-tion. Crit. Care Med. 2018, 46, 1923-1933. [CrossRef] [PubMed]

20. Force, A.D.T.; Ranieri, V.M.; Rubenfeld, G.D.; Thompson, B.; Ferguson, N.; Caldwell, E. Acute respiratory distress syndrome. JAMA 2012, 307, 2526-2533. 
21. Simonnet, A.; Chetboun, M.; Poissy, J.; Raverdy, V.; Noulette, J.; Duhamel, A.; Labreuche, J.; Mathieu, D.; Pattou, F.; Jourdain, M.; et al. High prevalence of obesity in severe acute respiratory syndrome coronavirus-2 (SARS-CoV-2) requiring invasive mechanical ventilation. Obesity 2020, 28, 1195-1199. [CrossRef]

22. Grasselli, G.; Zangrillo, A.; Zanella, A.; Antonelli, M.; Cabrini, L.; Castelli, A.; Cereda, D.; Coluccello, A.; Foti, G.; Fumagalli, R.; et al. Baseline characteristics and outcomes of 1591 patients infected with SARS-CoV-2 admitted to ICUs of the Lombardy Region, Italy. JAMA 2020, 323, 1574-1581. [CrossRef] [PubMed]

23. Guan, W.J.; Liang, W.H.; Zhao, Y.; Liang, H.-R.; Chen, Z.-S.; Li, Y.-M.; Liu, X.-Q.; Chen, R.-C.; Tang, C.-L.; Wang, T.; et al. Comorbidity and its impact on 1590 patients with COVID-19 in China: A nationwide analysis. Eur. Respir. J. 2020, 55, 2000547. [CrossRef]

24. Williams, B.; Mancia, G.; Spiering, W.; Agabiti Rosei, E.; Azizi, M.; Burnier, M.; Clement, D.L.; Coca, A.; De Simone, G.; Dominiczak, A.; et al. 2018 ESC/ESH Guidelines for the management of arterial hypertension: The task force for the management of arterial hypertension of the European Society of Cardiology (ESC) and the European Society of Hy-pertension (ESH). Eur. Heart J. 2018, 39, 3021-3104. [CrossRef]

25. Richardson, S.; Hirsch, J.S.; Narasimhan, M.; Crawford, J.M.; McGinn, T.; Davidson, K.W.; Barnaby, D.P.; Becker, L.B.; Chelico, J.D.; Cohen, S.L.; et al. Presenting characteristics, comorbidities, and outcomes among 5700 patients hospitalized with COVID-19 in the New York City Area. JAMA 2020, 323, 2052-2059. [CrossRef]

26. Yang, J.; Zheng, Y.; Gou, X.; Pu, K.; Chen, Z.; Guo, Q.; Ji, R.; Wang, H.; Wang, Y.; Zhou, Y. Prevalence of comorbidities and its effects in patients infected with SARS-CoV-2: A systematic review and meta-analysis. Int. J. Infect. Dis. 2020, 94, 91-95. [CrossRef] [PubMed]

27. Zhou, F.; Yu, T.; Du, R.; Fan, G.; Liu, Y.; Liu, Z.; Xiang, J.; Wang, Y.; Song, B.; Gu, X.; et al. Clinical course and risk factors for mortality of adult inpatients with COVID-19 in Wuhan, China: A retrospective cohort study. Lancet 2020, 395, 1054-1062. [CrossRef]

28. Paton, J.F.R.; Felippe, I.; Paterson, D.J.; Donnelly, J. What Should We Add to the Intensivists Mask to Relieve "Hypoxic Happiness" in COVID-19 Patients? The Physiological Society. Available online: https:/ /www.physoc.org/blog/hypoxic-happiness-covid19/ (accessed on 14 September 2020).

29. Velavan, T.P.; Meyer, C.G. Mild versus severe COVID-19: Laboratory markers. Int. J. Infect. Dis. 2020, 95, 304-307. [CrossRef] [PubMed]

30. Mueller, A.A.; Tamura, T.; Crowley, C.P.; DeGrado, J.R.; Haider, H.; Jezmir, J.L.; Keras, G.; Penn, E.H.; Massaro, A.F.; Kim, E.Y. Inflammatory biomarker trends predict respiratory decline in COVID-19 patients. Cell Rep. Med. 2020, 1, 100144. [CrossRef] [PubMed]

31. Bertin, D.; Brodovitch, A.; Beziane, A.; Hug, S.; Bouamri, A.; Mege, J.L.; Heim, X.; Bardin, N. Anticardiolipin IgG autoantibody level is an independent risk factor for COVID-19 severity. Arthritis Rheumatol. 2020, 72, 1953-1955. [CrossRef] [PubMed]

32. Hoeboer, S.H.; Straaten, H.M.O.-V.; Groeneveld, A.B.J. Albumin rather than C-reactive protein may be valuable in predicting and monitoring the severity and course of acute respiratory distress syndrome in critically ill patients with or at risk for the syndrome after new onset fever. BMC Pulm. Med. 2015, 15, 1-13. [CrossRef] [PubMed]

33. Huang, J.; Cheng, A.; Kumar, R.; Fang, Y.; Chen, G.; Zhu, Y.; Lin, S. Hypoalbuminemia predicts the outcome of COVID-19 independent of age and co-morbidity. J. Med. Virol. 2020, 92, 2152-2158. [CrossRef]

34. Ritter, M.; Ott, D.V.M.; Paul, F.; Haynes, J.-D.; Ritter, K. COVID-19: A simple statistical model for predicting intensive care unit load in exponential phases of the disease. Sci. Rep. 2021, 11, 1-12. [CrossRef]

35. Heo, J.; Han, D.; Kim, H.J.; Kim, D.; Lee, Y.K.; Lim, D.; Hong, S.O.; Park, M.J.; Ha, B.; Seog, W. Prediction of patients requiring intensive care for COVID-19: Development and validation of an integer-based score using data from Centers for Disease Control and Pre-vention of South Korea. J. Intensive Care 2021, 29, 16. [CrossRef] 